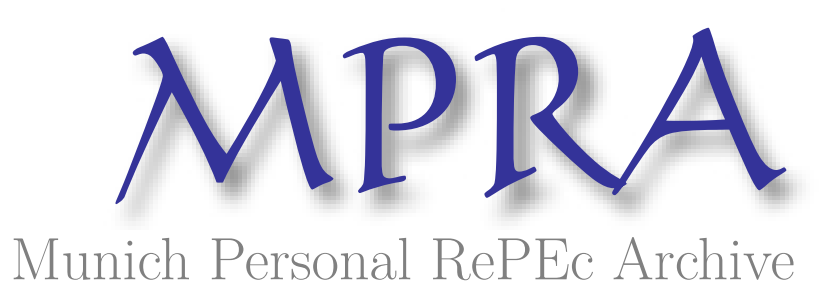

\title{
Estimating Production Functions with Fixed Effects
}

\author{
Abito, Jose Miguel \\ University of Pennsylvania
}

24 December 2019

Online at https://mpra.ub.uni-muenchen.de/97825/

MPRA Paper No. 97825, posted 08 Jan 2020 09:46 UTC 


\title{
Estimating Production Functions with Fixed Effects*
}

\author{
Jose Miguel Abito
}

December 24, 2019

\section{PRELIMINARY DRAFT. COMMENTS ARE GREATLY APPRECIATED}

\begin{abstract}
I propose an estimation procedure that can accommodate fixed effects in the widely used proxy variable approach to estimating production functions. The procedure allows unobserved productivity to have a permanent component in addition to a (nonlinear) Markov shock. The procedure does not rely on differencing out the fixed effect and thus is not restricted to within-firm variation for identification. Finally, the procedure is easy to implement as it only entails adding a two stage least squares using internal instruments.
\end{abstract}

*Abito: University of Pennsylvania, Wharton School, Business Economics and Public Policy, abito@wharton.upenn.edu. 


\section{Introduction}

Estimation of production functions is a staple in several fields in economics including agriculture and resource economics, trade, macroeconomics, and industrial organization, to name a few. An important econometric problem that has spurred a "search for identification" (Griliches and Mairesse, 1998) is the problem of simultaneity. (Marschak and Andrews, 1944). Observed input choices of firms are not under the control of the econometrician but instead reflect optimal behavior of the firm. Thus, the relationship between inputs and output may depend on factors that are observed by the firm but not by the econometrician. Not accounting for these unobserved factors - often referred to as unobserved productivitycreates bias in estimates of the production function.

Different methods essentially impose different assumptions on unobserved productivity, with the econometrician facing a tradeoff in choosing the method most appropriate for the data and institutional setting. In this paper, I develop an estimation procedure that combines the strengths of the two most popular estimation methods and thus relaxes the tradeoff between the two. Specifically, just as in the dynamic panel approach (Arellano and Bond, 1995; Blundell and Bond, 1998, 2000), I allow unobserved productivity to have both a permanent component (i.e. a fixed effect) and a time-varying component that follows a Markov process. However, I do not restrict the Markov process to be linear and the estimation method does not require differencing of the fixed effect. To do this, I adopt the assumption

of an existence of a proxy variable that provides an expression of unobserved productivity as a function of observables. Thus, one can view the estimation method in the paper as an extension of proxy variable methods (Olley and Pakes, 1996; Levinsohn and Petrin, 2003; Ackerberg, Caves and Frazer, 2015) that is robust to inclusion of a fixed effect in unobserved productivity. In fact, estimation of capital and labor output elasticities only involves adding a two stage least squares step to the standard proxy variable estimation procedure. More- 
over, the estimation procedure does not require additional proxies for instruments. Instead, "internal" instruments are constructed from the control function based on a single proxy variable. Therefore the method is readily accessible to econometricians who are already planning to use some form of the proxy variable approach.

A robust observation about productivity is that it is persistent and exhibits large dispersion even in narrowly defined industries (see Syverson (2011)). It seems natural to use methods that are robust to inclusion of a fixed effect. Current methods rely on differencing out fixed which can be subject to attenuation bias (Griliches and Hausman, 1986) and often exhibit poor finite sample performance (Blundell and Bond, 1998; Blundell and Bond, 2000).

The method I propose does not rely on differencing. Instead I construct instruments from the data that are orthogonal to the fixed effect. This strategy is similar to the additional moment conditions suggested by Blundell and Bond (1998, 2000) which uses the level equation with first differences of inputs as instruments. However, instead of relying on an auxiliary assumption that the fixed effect is orthogonal to the growth in inputs, which are endogenous decision variables of the firm, orthogonality is between time-varying productivity shocks and the fixed effect, both of which are exogenous in the model.

The proxy variable approach assumes that there exists a strictly monotonic mapping between unobserved productivity and a firm's decision variable (the proxy). The econometrician can then invert this mapping to express unobserved productivity as a function of observables, including the proxy variable. There are however two reasons why the proxy variable approach, as is often formulated, cannot accommodation a permanent fixed component in unobserved productivity. First, as Ackerberg, Caves and Frazer (2015) and Ackerbeg (2016) show, the proxy variable approach essentially rely on timing assumptions for identification. Basically the econometrician exploits the gap between when an action is taken and when unobservables (to the econometrician) such as productivity enter the firm's information 
set. When unobserved productivity includes a permanent component such as a fixed effect, then all actions will be correlated with the unobservable. To the best of my knowledge, there is currently no method that allows this case without sacrificing the attractive features of proxy variable methods. ${ }^{1}$

Second, if unobserved productivity also includes a fixed effect, then the inversion may fail. This is known as the scalar unobservable assumption. Similar to an extension to Gandhi, Navarro and Rivers (2017, Appendix O5-1), I assume that there is a proxy that it is a strictly increasing function of the sum of the two components of unobserved productivity. Although the two components cannot be separately expressed as functions of observables, one can still invert the relationship to express the sum of the components as a function of observables.

The key step then is to think about the sum of the two components of unobserved productivity as having a measurement error form: the latent variable is the time-varying Markov component while the fixed component is the measurement error. This measurement error is not classical since it is correlated with input choices including other potential proxy variables. Thus, I cannot use double measurements such as multiple proxies subject to independent measurement errors as instruments (e.g. Hu, Huang and Sasaki, (2019)).

Instead, I rely on a different instrumental variable strategy that uses a Berskon instrument: the measurement error is correlated with the latent variable, but independent of the instrument (Schennach, 2007). I construct Berkson instruments by taking first differences

\footnotetext{
${ }^{1}$ Gandhi, Rivers and Navarro (2017) allow for a fixed effect as an extension of their estimation procedure. However, they assume that the Markov component evolves as a linear $(\mathrm{AR}(1))$ process and rely on the same differencing technique as in the dynamic panel approach.

More recently, Lee, Stoyanov and Zubanov (2019) consider a model where a fixed effect enters the evolution of productivity, i.e. $\omega_{i t}=g\left(\omega_{i t-1}\right)+c_{i}+\xi_{i t}$ assuming the inversion of the proxy variable gives $\omega_{i t}$. This is similar to the set up in Theorem 1 of Asker, Collard-Wexler and De Loecker (2014) whereby the fixed effect eventually appears linearly in the estimating equation which can then be differenced away.
} 
of the inverted function involving the proxy variable. Given this instrument, I show how to estimate the labor and capital coefficients by adding a two stage least squares step.

Using Monte Carlo experiments, I compare the performance of the proposed estimation procedure with some of the widely used estimators in the literature. Proxy variable approaches yield inconsistent estimators when there is a fixed effect hence the bias persists even in relativity large samples. In contrast, the estimation procedure that uses a Berkson instrument is robust to inclusion of the fixed effect and performs as well as the standard proxy variable approach when there is no fixed effect. Compared to methods that rely on differencing, the results point to better finite sample performance in terms of bias and precision. Finally, the system GMM approach (Arellano and Bover, 1995; Blundell and Bond, 1998, 2000) only works if the fixed effect is orthogonal to the growth in inputs. Since the growth in capital is driven by investment, this essentially requires that the way investment affects growth is orthogonal to a state variable such as a fixed effect. Indeed I do observe biased estimates when the role of the fixed effect in determining investment is larger.

The paper is related to the literature that relaxes the scalar unobservable assumption in proxy variable methods, specifically to the strand of the literature that develops methods to handle measurement error in inputs and proxies. Hu, Huang and Sasaki (2019) establish identification of such a model using results from the nonlinear errors-in-variables literature (i.e. Hu and Schennach (2008)). Their strategy is to use multiple proxies, which they assume as having independent measurement errors. In contrast to this double measurement strategy, I use an instrumental variables strategy with a Berkson instrument (Schennach, 2007). Finally, Kim, Petrin and Song (2016) and Collard-Wexler and De Loecker (2017) both focus on the case where capital is measured with error. Similar to $\mathrm{Hu}$, Huang and Sasaki (2019), Kim, Petrin and Song (2016) use results form the nonlinear errors-in-variables literature and suggest an estimation procedure based on sieves. In contrast, Collard-Wexler 
Table 1: Abbreviations of key papers cited

\begin{tabular}{l|l}
\hline Ackerberg, Caves and Frazer (2015) & ACF \\
Blundell and Bond (1998) & BB98 \\
Blundell and Bond (2000) & BB00 \\
Gandhi, Rivers and Navarro (2017) & GNR \\
Levinsohn and Petrin (2003) & LP \\
Olley and Pakes (1996) & OP \\
\hline
\end{tabular}

and De Loecker (2017) leverage on a Cobb Douglas production function to pose the problem as a linear errors-in-variables model, and use investment as an instrument for mismeasured capital. They emphasize the attractiveness of methods that are easy to implement and readily accessible.

The next section presents the model and key assumptions maintained throughout the paper. Section 3 discusses both identification and estimation. Section 4 contains the Monte Carlo experiments and finally section 5 concludes. For ease of exposition, Table 1 lists the abbreviations of some of the key papers I repeatedly refer to.

\section{Model}

Consider the following production function: ${ }^{2}$

$$
y_{i t}=\beta_{l} l_{i t}+\beta_{k} k_{i t}+w_{i t}+\varepsilon_{i t} .
$$

\footnotetext{
${ }^{2}$ I discuss the approach in the context of value-added production functions to keep the discussion as close to OP, LP and ACF.
} 
The variable $y_{i t}$ is firm $i$ 's log output at time $t$, and $l_{i t}$ and $k_{i t}$ are the labor and capital inputs (in logs) respectively. These inputs and output are observed by the econometrician. In contrast, the econometrician does not observe $\varepsilon_{i t}$ and $w_{i t}$. The unobservable $\varepsilon_{i t}$ represents shocks to the production function that are also unobserved by the firm at the time it decides on its time $t$ inputs. This unobservable can represent measurement error in output or other factors that affect output (holding inputs fixed) that the firm cannot predict. On the other hand, $w_{i t}$ represents factors that are observed (or predicted) by the firm and taken into account in its input choices. The unobservable $w_{i t}$ is often thought of as a firm's "productivity" which is observed by the firm but not by the econometrician.

The goal of the econometrician is to estimate parameters $\beta_{l}$ and $\beta_{k}$, which are the output elasticities for labor and capital respectively. In this paper, I focus on the problem of simultaneity (Marschak and Andrews, 1944). Since the firm takes productivity $w_{i t}$ into account when choosing its inputs, an OLS regression of output on inputs will generate biased estimates.

As a solution to the simultaneity problem, the proxy variable approach (e.g. OP, LP and ACF) exploits a "structural" function that maps a firm's productivity to an endogenous decision. In OP, investment is assumed to be strictly increasing in productivity and thus can be inverted to express it as a function of observables (e.g. investment, capital stock and age). That is, if $I_{i t}$ refers to investment and

$$
I_{i t}=f\left(w_{i t}, k_{i t}, a g e_{i t}\right)
$$

where $f$ is strictly increasing in $\omega_{i t}$, then

$$
\left.w_{i t}=f^{-1}\left(I_{i t}, k_{i t}, \operatorname{age}_{i t}\right)\right)=h\left(x_{i t}\right)
$$

where $x_{i t}=\left(I_{i t}, k_{i t}, a g e_{i t}\right)$. LP suggest using intermediate inputs as a proxy variable since data usually contain a lot of observations with zero investment. As in OP, LP assumes 
that the intermediate input is a strictly increasing function of productivity, and thus can be inverted to control for the latter.

An important assumption in proxy variable methods is that unobserved productivity evolves as a Markov process. Formally, unobserved productivity $w_{i t}$ is assumed to be equal to

$$
\omega_{i t}=E\left[\omega_{i t} \mid \mathcal{I}_{i t-1}\right]=E\left[\omega_{i t} \mid \omega_{i t-1}\right]+\xi_{i t}=g\left(\omega_{i t-1}\right)+\xi_{i t} .
$$

where $\mathcal{I}_{i t-1}$ is firm $i$ 's information set at time $t-1$. This Markov assumption implies $E\left[\xi_{i t} \mid \mathcal{I}_{i t-1}\right]=0$, and thus one can generate moment conditions based on the timing of decisions and the information used when making these decisions (ACF; Ackerberg, 2016). For example, since past decisions on the labor input belongs to $\mathcal{I}_{i t-1}$, then $E\left[\xi_{i t} \mid l_{i t-j}\right]=0$ for all $j \geq 1$. These timing assumptions are powerful since not only do they provide moment conditions for estimation, they also allow the econometrician to generate and use "internal" instruments, e.g. lagged input choices.

The timing assumption breaks down when unobserved productivity has a permanent component. To see this, suppose $w_{i t}=\omega_{i t}+a_{i}$ where $\omega_{i t}$ satisfies the Markov assumption as above. Although $E\left[\xi_{i t} \mid \mathcal{I}_{i t-1}\right]=0$ remains valid, $E\left[\xi_{i t}+a_{i} \mid \mathcal{I}_{i t-1}\right]=0$ is not. In fact, when unobserved productivity has a permanent component, all input choices will be correlated with $a_{i}$ regardless of how far in the past the decision was made.

One potential solution is to eliminate $a_{i}$ from the estimating moment conditions. This is possible under the proxy variable approach if either $a_{i}$ does not enter the proxy equation, or if it does, $g(\cdot)$ is linear (GNR). If $a_{i}$ does not enter the proxy equation, then $\omega_{i t-1}=h\left(x_{i t-1}\right)$ and we can rewrite the production function as

$$
y_{i t}=\beta_{l} l_{i t}+\beta_{k} k_{i t}+g\left(h\left(x_{i t-1}\right)\right)+\left(\xi_{i t}+a_{i}+\varepsilon_{i t}\right)
$$

We can then eliminate $a_{i}$ from the estimating equation by taking first differences. 
Suppose instead that $a_{i}$ enters the proxy variable equation. This violates the so-called scalar unobservable assumption hence one can no longer express $\omega_{i t-1}$ as a function of only observables. In Appendix O5-1 of GNR, they extend their model to the case where unobserved productivity has a fixed effect component. They assume that the proxy variable is a strictly increasing function of the sum $\left(\omega_{i t}+a_{i}\right)$, and thus $h\left(x_{i t}\right)=\omega_{i t}+a_{i}$ in my notation. Such an assumption is tenable if the proxy is static, otherwise the proxy is likely to be a function of the state variables $\omega_{i t}$ and $a_{i}$ separately, and not only their sum (Ackerberg, 2016). Following GNR, suppose this assumption holds, e.g. we use intermediate inputs that are assumed to be static, the production function becomes

$$
y_{i t}=\beta_{l} l_{i t}+\beta_{k} k_{i t}+g\left(h\left(x_{i t-1}\right)-a_{i}\right)+\left(\xi_{i t}+a_{i}+\varepsilon_{i t}\right) .
$$

In this case, we need $g(\cdot)$ to be linear in order to difference out $a_{i}$, similar to the dynamic panel approach (Arellano and Bond, 1991; BB98; BB00). A drawback of the dynamic panel approach is that identification relies on within-firm variation, which may not be a good source of variation when output and inputs are highly serially correlated (Griliches and Hausman, 1986; BB98). ${ }^{3}$

In the next section, I show how to identify and estimate output elasticities in a model that largely follows the proxy variable approach but allows for a fixed effect in unobserved productivity. Unless stated otherwise, I maintain the following assumptions throughout the paper:

\section{Unobserved productivity:}

$$
w_{i t}=\omega_{i t}+a_{i}
$$

\footnotetext{
${ }^{3}$ GNR (Table O6.5) find lower and noisier capital estimates for Colombia using an extension of their method that allows for fixed effects.
} 
2. Markov:

$$
\omega_{i t}=E\left[\omega_{i t} \mid \mathcal{I}_{i t-1}\right]+\xi_{i t}=g\left(\omega_{i t-1}\right)+\xi_{i t}
$$

where $g(\cdot)$ can be nonlinear.

\section{Timing:}

$$
E\left[\xi_{i t} \mid k_{i t-j}\right]=0 \text { for all } j \geq 0
$$

\section{Proxy variable:}

$$
\omega_{i t}+a_{i}=h\left(x_{i t}\right)
$$

where $x_{i t}$ is a vector of observables including the proxy variable.

\section{Mean independence:}

$$
E\left[a_{i} \mid \xi_{i t}\right]=0 \text { for all } t
$$

The first assumption assumes the same error structure as in BB00 and GNR. The second and third assumptions are standard in the proxy variable literature, while the fourth assumption follows GNR as discussed above. Finally the fifth assumption consists of normalizing the mean of the fixed effect to zero, and also the assumption that unexpected innovations $\xi_{i t}$ are not informative of $a_{i}$. Note that mean independence is sufficient as opposed to full independence. The purpose of the fifth assumption will be clearer in the next section.

\section{Identification and estimation}

Using the previous assumptions, we can rewrite the production function as

$$
y_{i t}=\beta_{l} l_{i t}+\beta_{k} k_{i t}+g\left(\omega_{i t-1}\right)+\left(\xi_{i t}+a_{i}+\varepsilon_{i t}\right)
$$

There are two issues in estimating the coeffcients $\beta_{l}$ and $\beta_{k}$. First, even if we observe $\omega_{i t-1}$, input choices (including the proxy) are still endogenous because $a_{i}$ is unobserved and 
correlated with the inputs. Second, $\omega_{i t-1}$ is unobserved and cannot be simply replaced by $h\left(x_{i t}\right)$ as in the standard proxy variable case where $a_{i}=0$.

To solve the first issue, I use a vector of instruments $z$ such that

$$
E\left[\xi_{i t}+a_{i}+\varepsilon_{i t} \mid z\right]=0 .
$$

Consider the decomposition

$$
\begin{aligned}
l_{i t} & =E\left[l_{i t} \mid z\right]+\eta_{l i t}=\widehat{l}_{i t}+\eta_{l i t} \\
k_{i t} & =E\left[k_{i t} \mid z\right]+\eta_{k i t}=\widehat{k}_{i t}+\eta_{k i t}
\end{aligned}
$$

where by construction, $E\left[\eta_{l i t} \mid z\right]=E\left[\eta_{k i t} \mid z\right]=0$. I can then rewrite the estimating equation as

$$
y_{i t}=\beta_{l} \widehat{l}_{i t}+\beta_{k} \widehat{k}_{i t}+g\left(\omega_{i t-1}\right)+\left(\beta_{l} \eta_{l i t}+\beta_{k} \eta_{k i t}+\xi_{i t}+a_{i}+\varepsilon_{i t}\right) .
$$

Thus, if $\omega_{i t-1}$ were observed, then one can estimate $\beta$ 's by applying OLS to equation 2 .

As for the second issue, although $\omega_{i t-1}$ is unobserved, we do observe the sum $\omega_{i t-1}+a_{i}$ up to some unknown function $h(\cdot)$ of observables:

$$
h\left(x_{i t-1}\right)=\omega_{i t-1}+a_{i} .
$$

We can think of $\omega_{i t-1}$ as a latent variable that we observe subject to some measurement error $a_{i}$. A common solution to handle nonlinear errors-in-variables models is to use a second $i n$ dependent measurement as an instrument. In the context of production function estimation, Hu, Huang and Sasaki (2019)), for example, assume the econometrician has multiple proxies with independent measurement errors. However in my case, the measurement error $a_{i}$ is potentially correlated with all proxies and inputs, and thus the strategy of using multiple proxies will not work.

Schennach (2007) establishes nonparametric identification of a nonlinear errors-in-variables model using an instrument that has a Berkson-error form (Berkson, 1950; Chen, Hong and 
Nekipelov, 2011). Instead of assuming independence between the latent variable and the measurement error, a measurement with Berkson-error satisfies the "causal equation"

$$
\omega_{i t-1}=m(z)+v
$$

where the instrument $z$ and the error $v$ are independent, $m(\cdot)$ is some function ${ }^{4}$, and $E\left[\xi_{i t}+\right.$ $\left.\varepsilon_{i t} \mid z, v\right]=0$ and $E\left[a_{i} \mid z, v, \xi_{i t}\right]=0$. In contrast to classical measurement error, the latent variable is correlated with the measurement error $v$. The independence assumption instead is between $m(z)$ and the measurement error. Given these assumptions, Schennach (2007) shows that $\beta_{l}, \beta_{k}$ and $g(\cdot)$ are identified. ${ }^{5}$

Nonlinear errors-in-variables models are often complicated to estimate and implementation is often still challenging (Collard-Wexler and De Loecker, 2016). It turns out that if we assume $g(\cdot)$ is a polynomial, then estimating the coefficients $\beta_{l}$ and $\beta_{k}$ is pretty straightforward. To show this, I rely on results from Hausman, Newey, Ichimura and Powell (1991), where they show identification using Berkson instruments when $g(\cdot)$ is a polynomial of known degree $P$ :

$$
g(\omega)=\sum_{j=0}^{P} \beta_{j} \omega^{j} .
$$

Under the polynomial assumption, the estimating equation (2) becomes

$$
y_{i t}=\beta_{l} \widehat{l}_{i t}+\beta_{k} \widehat{k}_{i t}+\sum_{j=0}^{P} \gamma_{j} \widehat{\omega}_{i t-1}^{j}+e_{i t}
$$

${ }^{4}$ The function $m(\cdot)$ is identified and can be estimated using data on $h\left(x_{i t-1}\right)$ and $z$. To see this note

$$
h\left(x_{i t-1}\right)=\omega_{i t-1}+a_{i}=m(z)+(v+a)
$$

where $E[v+a \mid z]=0$ by assumption.

${ }^{5}$ Schennach's (2007) main model does not include regressors such as $l$ and $k$. A model similar to equation 2 is mentioned as a simple extension. See Schennach (2007, p. 222). 
where $\widehat{\omega}_{i t-1}=m(z)$,

$$
\gamma_{j} \equiv\left(\sum_{q=j}^{P} \beta_{j}\left(\begin{array}{l}
q \\
j
\end{array}\right) E\left(v_{i t}^{q}\right)\right)
$$

and

$$
e_{i t} \equiv \sum_{j=0}^{P} \widehat{\omega}_{i t-1}^{j}\left(\sum_{q=j}^{P} \beta_{j}\left(\begin{array}{l}
q \\
j
\end{array}\right)\left[v_{i t}^{q}-E\left(v_{i t}^{q}\right)\right]\right)+\left(\beta_{l} \eta_{l i t}+\beta_{k} \eta_{k i t}+\xi_{i t}+a_{i}+\varepsilon_{i t}\right) .
$$

As long as the instrument vector $z$ satisfies $E\left[e_{i t} \mid z\right]=0$, then $\beta_{l}$ and $\beta_{k}$ can be identified using the moment condition

$$
E\left[e_{i t} \mid \widehat{l}_{i t}, \widehat{k}_{i t}, \widehat{\omega}_{i t-1}\right]=0 .
$$

Therefore we can estimate the output elasticities by running an OLS regression of $y_{i t}$ on $\widehat{l}_{i t}$, $\widehat{k}_{i t}$, and powers of $\widehat{\omega}_{i t-1}$.

\subsection{Internal Berkson instruments}

The feasibility of the previous identification strategy hinges on the existence of a set of instruments that satisfy the properties of a Berkson instrument. In the context of our model, we need

1. $E\left[\eta_{l i t} \mid z\right]=E\left[\eta_{k i t} \mid z\right]=0$,

2. $E\left[\xi_{i t}+a_{i}+\varepsilon_{i t} \mid z\right]=0$, and

3. $E\left[v_{i t}^{q} \mid z\right]=E\left[v_{i t}^{q}\right]$ for all $q=1,2, \ldots, P$.

The first condition follows by construction since

$$
\eta_{l i t}=l_{i t}-E\left[l_{i t} \mid z\right] \text { and } \eta_{k i t}=k_{i t}-E\left[k_{i t} \mid z\right]
$$

and therefore I focus on the second and third conditions. 
I propose using changes in unobserved productivity and its lags as instruments:

$$
\Delta \omega_{i t-j}=\Delta h\left(x_{i t-j}\right)
$$

for $j \geq 1$. The advantage of these instruments is that they are "internal" in that they can be constructed from the data at hand. These instruments are similar to the instruments proposed by Blundell and Bond $(1998,2000)$ to improve the finite sample performance of the dynamic panel approach. There, a crucial assumption is that changes in endogenous variables, i.e. $\Delta l_{i t}$ and $\Delta k_{i t}$. are mean independent with $a_{i}$. In my case, mean independence is between $a_{i}$ and a function of $\xi_{i t-j}$. In addition, since $E\left[\xi_{i t} \mid \mathcal{I}_{i t-1}\right]=0$ and $\Delta \omega_{i t-j} \in \mathcal{I}_{i t-1}$, then indeed $E\left[\xi_{i t}+a_{i}+\varepsilon_{i t} \mid \Delta \omega_{i t-j}\right]=0$.

Finally, we need the instruments to satisfy $E\left[v_{i t}^{q} \mid z\right]=E\left[v_{i t}^{q}\right]$ for all $q=1,2, \ldots, P$. This is implied by the structure of the Berskon-error, i.e. $z(m(z))$ is independent of

$$
v=\omega_{i t-1}-m(z)
$$

Mean independence between $z$ and $v$ is satisfied by construction since I estimate $m(z)=$ $E\left[h\left(x_{i t-1}\right) \mid z\right]=E\left[\omega_{i t-1} \mid z\right]$. What is then needed is mean independence between $z$ and higher moments of $v$ (up to the $P$-th moment). ${ }^{6}$

\subsection{Comparison of identifying moment conditions}

Following Ackerberg (2016), consider a model where $g(\cdot)$ is linear and $\varepsilon=0$. The goal of this section is to compare the moment conditions that identify the parameters from the proposed estimation procedure with the proxy variable and dynamic panel approaches.

\footnotetext{
${ }^{6}$ If $g(\cdot)$ is linear, then mean independence between $z$ and $v$ is sufficient. Also, if we observe all lags of $\Delta \omega_{i t-j}$, i.e. $z=\left(\Delta \omega_{i t-1}, \Delta \omega_{i t-2, \ldots, \Delta \omega_{i 0}}\right)$, then we essentially observe $\omega_{i t-1}$ and $m(z)$ and $v$ are trivially independent.
} 
Under the given simplifications, the identifying moment condition using the proposed estimation strategy is

$$
E\left[\left(y_{i t}-\beta_{l} l_{i t}-\beta_{k} k_{i t}-\rho h\left(x_{i t-1}\right)\right) \cdot\left(\begin{array}{c}
\Delta \omega_{i t-1} \\
\Delta \omega_{i t-2} \\
\Delta \omega_{i t-3}
\end{array}\right)\right]=\mathbf{0}
$$

This moment condition is the same as the moment condition in ACF (Ackerberg's (2016) equation 5) except that the instrument vector in ACF is $z=\left(h\left(x_{i t-1}\right), l_{i t}, k_{i t}\right)$. Since $y_{i t}-$ $\beta_{l} l_{i t}-\beta_{k} k_{i t}-\rho h\left(x_{i t-1}\right)=\xi_{i t}+(1-\rho) a_{i}$ and

$$
E\left[a_{i} \cdot\left(\begin{array}{c}
h\left(x_{i t-1}\right) \\
l_{i t} \\
k_{i t}
\end{array}\right)\right] \neq \mathbf{0}
$$

the moment condition in ACF no longer holds unless $a_{i}=0$. In contrast, the instrument vector in equation 4 has $a_{i}$ differenced out.

The moment condition for the dynamic panel approach is given by

$$
E\left[\left(\Delta \Delta_{\rho} y_{i t}-\beta_{l} \Delta \Delta_{\rho} l_{i t}-\beta_{k} \Delta \Delta_{\rho} k_{i t}\right) \cdot\left(\begin{array}{c}
l_{i t-3} \\
k_{i t-2} \\
y_{i t-3}
\end{array}\right)\right]=0
$$

where, for example, $\Delta \Delta_{\rho} y_{i t}=\left(y_{i t}-\rho y_{i t-1}\right)-\left(y_{i t-1}-\rho y_{i t-2}\right)$. This moment condition is valid since $a_{i}$ is differenced away. However, BB98 find that this moment condition leads to poor finite sample properties (i.e. biased and imprecise estimates) due to weak identification. If one is willing to assume the assumption

$$
E\left[a_{i} \mid \Delta l_{i t}, \Delta k_{i t}\right]=0,
$$


then they suggest adding the following moment condition:

$$
E\left[\left(\Delta_{\rho} y_{i t}-\beta_{l} \Delta_{\rho} l_{i t}-\beta_{k} \Delta_{\rho} k_{i t}\right) \cdot\left(\begin{array}{c}
\Delta l_{i t-1} \\
\Delta k_{i t}
\end{array}\right)\right]=0 .
$$

The estimator based on moment conditions 5 and 6 is sometimes referred to as the "system GMM" estimator.

Observe that

$$
\Delta_{\rho} y_{i t}-\beta_{l} \Delta_{\rho} l_{i t}-\beta_{k} \Delta_{\rho} k_{i t}=y_{i t}-\beta_{l} l_{i t}-\beta_{k} k_{i t}-\rho\left(y_{i t-1}-\beta_{l} l_{i t-1}-\beta_{k} k_{i t-1}\right) .
$$

Since in a model without $\varepsilon$,

$$
h\left(x_{i t-}\right)=y_{i t-1}-y_{i t-1}-\beta_{l} l_{i t-1}-\beta_{k} k_{i t-1},
$$

the additional moment used in the system GMM estimator can be rewritten as

$$
E\left[\left(y_{i t}-\beta_{l} l_{i t}-\beta_{k} k_{i t}-\rho h\left(x_{i t-1}\right)\right) \cdot\left(\begin{array}{c}
\Delta l_{i t-1} \\
\Delta k_{i t}
\end{array}\right)\right]=0 .
$$

But this is the same as moment condition 4, except for the choice of instruments. Note though that both sets of instrument essentially differences out the fixed effect $a_{i}$. Therefore, one can view the proposed estimation procedure as combining the assumption used in the system GMM estimator, with the proxy variable assumption that allows us to construct the instruments $\Delta h\left(x_{i t-j}\right)=\Delta \omega_{i t-j}$.

\subsection{Estimation}

I now discuss estimation of the model under the assumption that $g(\cdot)$ is a polynomial of known degree as in Hausman, Newey, Ichimura and Powell (1991). The estimation procedure is iterative and proceeds in several steps. 
Step 0 is basically the first stage in OP, LP or ACF. That is, I get an estimate $\widehat{\Phi}_{i t}$ of

$$
\Phi_{i t}\left(l_{i t}, k_{i t}, x_{i t}\right)=\beta_{l} l_{i t}+\beta_{k} k_{i t}+h\left(x_{i t}\right)
$$

by estimating the partial linear model

$$
y_{i t}=\Phi_{i t}\left(l_{i t}, k_{i t}, x_{i t}\right)+\varepsilon_{i t}
$$

Step 1 is the start of the iteration. Given a guess $\left(\widetilde{\beta}_{l}, \widetilde{\beta}_{k}\right)$ and the estimate $\widehat{\Phi}_{i t}$ from Step 0, construct instruments:

$$
\Delta h\left(x_{i t-j}\right)=\Delta \widehat{\Phi}_{i t-j}-\widetilde{\beta}_{l} \Delta l_{i t-j}-\widetilde{\beta}_{k} \Delta k_{i t-j}
$$

for $j \geq 1$. We need to generate at least three instruments (three lags) to be (exactly) identified.

Once the instruments are constructed, we proceed to Step 2 which can be thought of as the first stage in two stage least squares. That is, we estimate the conditional expectations

$$
\begin{aligned}
\widehat{l}_{i t} & =E\left[l_{i t} \mid z\right] \\
\widehat{k}_{i t} & =E\left[k_{i t} \mid z\right] \\
\widehat{\omega}_{i t-1} & =E\left[h\left(x_{i t-1}\right) \mid z\right]
\end{aligned}
$$

where $z$ is the vector of instruments, e.g. $z=\left(\Delta h\left(x_{i t-1}\right), \Delta h\left(x_{i t-2}\right), \Delta h\left(x_{i t-3}\right)\right)$.

Step 3 then is just an OLS regression of $y_{i t}$ on $\widehat{l}_{i t}, \widehat{k}_{i t}$ and powers of $\widehat{\omega}_{i t}$. This will give estimates $\widehat{\beta}_{l}\left(\widetilde{\beta}_{l}, \widetilde{\beta}_{k}\right)$ and $\widehat{\beta}_{k}\left(\widetilde{\beta}_{l}, \widetilde{\beta}_{k}\right)$. We repeat Steps 1 to 3 until we find the fixed point:

$$
\widetilde{\beta}_{l}=\widehat{\beta}_{l}\left(\widetilde{\beta}_{l}, \widetilde{\beta}_{k}\right) \text { and } \widetilde{\beta}_{k}=\widehat{\beta}_{k}\left(\widetilde{\beta}_{l}, \widetilde{\beta}_{k}\right) .
$$

For OP or LP, the proxy is not a function of $l_{i t}$ (i.e. $l_{i t}$ has independent variation), and so $\Phi_{i t}\left(k_{i t}, x_{i t}\right)=\beta_{k} k_{i t}+h\left(x_{i t}\right)$ and $\beta_{l}$ can be separately identified in Step 0. Moreover, we only need a guess $\widetilde{\beta}_{k}$ and two lags of the instrument to be exactly identified. 


\section{Monte Carlo experiments}

I perform Monte Carlo experiments to compare the finite sample performance of the proposed estimation procedure. To simplify the simulations, I assume a data generating process where LP is identified and consistent if $a_{i}=0$. Specifically, I assume log labor (in reduced form) is given by

$$
l_{i t}=\omega_{i t}+a_{i}+u_{i t}
$$

where $u_{i t}$ is iid and distributed standard normal. I also assume reduced forms for investment and $\log$ materials (i.e. the proxy):

$$
I_{i t}=\exp \left(0.1 \omega_{i t}+a_{i}+k_{i t}\right)
$$

and

$$
m_{i t}=\omega_{i t}+a_{i}+k_{i t}
$$

respectively. Finally, the capital accumulation equation is $K_{i t}=0.95 K_{i t-1}+I_{i t-1}$ where initial $\log$ capital, $\log K_{i 0}$, is a random draw from a standard normal distribution.

To generate productivity, I assume the fixed component $a_{i}$ is distributed iid standard normal. For the Markov component, I consider both linear and nonlinear processes:

$$
\omega_{i t}=\rho \omega_{i t-1}+\xi_{i t}
$$

and

$$
\omega_{i t}=\rho\left(\omega_{i t-1}-0.01 \omega_{i t-1}^{3}\right)+\xi_{i t}
$$

where $\rho \in\{0.2,0.8\}, \xi_{i t}$ is iid standard normal, and $\omega_{i 0}=0$.

I run 1000 replications, with $N=250$ firms and $T=5$ time periods. I estimate the labor and capital coefficients using OLS, first differencing (FE), the dynamic panel approach with and without the stationarity assumption (DPS and DP respectively), LP, and the approach proposed in the paper (proxy + Berkson IV, or PIV). 
Table 2 contains the results for $\rho=0.2$ and $g(\cdot)$ is linear. Table 3 corresponds to the case where $g(\cdot)$ is nonlinear. The first three columns of numbers are the mean, standard deviation and root mean squared errors over 1000 simulation runs when productivity does not have a fixed effect, while the next three is when productivity is equal to $\omega_{i t}+a_{i}$. Tables 4 and 5 instead contain the results for $\rho=0.8$. For all of these tables, the true values are $\beta_{l}=0.7$ and $\beta_{k}=0.3$.

Focusing on the OLS estimates, the bias is larger for $\beta_{l}$ compared to $\beta_{k}$. This observation seems to apply to the other estimation procedures as well, except for FE. The capital coefficient under FE is severely downward biased, consistent with what have been observed in the literature (e.g. Griliches and Hausman (1986), and the famous quote from Griliches and Mairesse (1998, p. 178)).

Consistent with BB98, the DP estimates are biased and are terribly imprecise. DPS on the other hand significantly improves the estimates. In fact, the estimate for the capital coefficient seem very reliable across specifications. However, even with DPS, the labor coefficient remains biased and imprecise in almost all specifications except for when there is no fixed effect, $\rho=0.8$ and $g(\cdot)$ is linear (Table 4$)$.

LP exhibits good finite sample properties in all specification with $a_{i}=0$. Since the simulation assumptions allow estimation of $\beta_{l}$ in the first stage, the labor coefficient does not suffer from bias even when there is a fixed effect. However, the capital coefficient is biased upwards when $a_{i} \neq 0$. The bias seems to get worse when $\rho$ is lower, i.e. the persistence in productivity (and other variables) are mostly due to the fixed component rather than the Markov component.

PIV performs well in all specifications. It is robust to inclusion of a fixed effect, and it does not suffer from a potential weak identification problem as in DP. Therefore PIV can be seen as taking advantage of both proxy variable and dynamic panel methods. 


\section{Conclusion}

In this paper, I show how one can allow for a fixed effect component in the widely used proxy variable method. There are two main benefits of the procedure. First, the procedure does not rely on differencing and also allows for a nonlinear Markov component. Second, the procedure is easy to implement as it only entails adding a two stage least squares step. Since the instruments used are constructed from functions of the proxy and its lags, the data requirements are the same as in most applications of proxy variable methods. I illustrate the performance of the estimaiton prcoedure using Monte Carlo simulations. The procedure performs as well as other existing estimation procedures in settings where assumptions of these estimators hold, and works better for settings where these estimators are known to fail. 


\section{References}

Ackerberg, D. A (2016), "Timing Assumptions and Efficiency: Empirical Evidence in a Production Function Context," working paper.

Ackerberg, D. A., K. Caves and G. Frazer (2015), "Identification Properties of Recent Production Function Estimators," Econometrica, 83:6, 2411-2451.

Arellano, M. and S. Bond (1991), "Some tests of specification for panel data: Monte Carlo evidence and an application to employment equations," Review of Economic Studies, $58: 2,277-297$.

Arellano, M. and O. Bover (1995), "Another Look at the Instrumental Variable Estimation of Error-Components Models," Journal of Econometrics 68:1, 29-51.

Asker, J. A. Collard-Wexler and J. De Loecker (2014), "Dynamic Inputs and Resource (Mis)Allocation," Journal of Political Economy, 122:5, 1013-1063.

Berkson, J. (1950), "Are There Two Regressions?" Journal of the American Statistical Association, 45:250, 164-180..

Blundell, R. and S. Bond (1998), "Initial Conditions and Moment Restrictions in Dynamic Panel Data Models," Journal of Econometrics, 87, 115-143.

Blundell, R. and S. Bond (2000), "GMM Estimation with persistent panel data: an application to production functions," Econometric Reviews, 19:3, 321-340.

Chen, X., H. Hong and D. Nekipelov (2011), "Nonlinear Models of Measurement Errors," Journal of Economic Literature, 49:4, 901-937. 
Collard-Wexler, A. and J. De Loecker (2016), "Production Function Estimation with Measurement Error in Inputs," working paper.

Gandhi, A., S. Navarro and D. Rivers, "On the Identification of Gross Output Production Functions," Journal of Political Economy, forthcoming.

Griliches, Z., and J. Hausman (1986), "Errors in Variables in Panel Data," Journal of Econometrics, 31, 93-118.

Griliches, Z., and J. Mairesse (1998), "Production Functions: The Search for Identification," in Econometrics and Economic Theory in the Twentieth Century: The Ragnar Frisch Centennial Symposium, ed. by S. Strm, Cambridge, U.K.: Cambridge University Press.

Hausman, J., W. Newey, H. Ichimura, and J. Powell (1991), "Measurement Errors in Polynomial Regression Models," Journal of Econometrics, 50, 273-295.

Hu, Y., G. Huang and Y. Sasaki (2019), "Estimating production functions with robustness against errors in the proxy variables," Journal of Econometrics, forthcoming.

Hu, Y. and S. Schennach (2008), "Instrumental Variable Treatment of Nonclassical Measurement Error Models," Econometrica, 76:1, 195-216.

Kim, K., A. Petrin, and S. Song (2016), "Estimating production functions with control functions when capital is measured with error," Journal of Econometrics, 190:2, 267279 .

Lee, Stoyanov and Zubanov (2019), "Olley and Pakes?style Production Function Estimators with Firm Fixed Effects," Oxford Bulletin of Economics and Statistics, 81:1, 79-97. 
Levinsohn, J. and A. Petrin (2003), "Estimating Production Functions Using Inputs to Control for Unobservables," Review of Economic Studies, 70:2, 317-341.

Marschak, J. and W. Andrews (1944), "Random Simultaneous Equations and the Theory of Production," Econometrica, 12:3/4, 143-205.

Olley, S. and A. Pakes (1996), "The Dynamics of Productivity in the Telecommunications Equipment Industry," Econometrica, 64:6, 1263-1297.

Schennach, S. M. (2007), "Instrumental Variable Estimation of Nonlinear Errors-in-Variables Models," Econometrica, 75:1, 201-239.

Syverson, C. (2011), "What Determines Productivity?" Journal of Economic Literature, 49:2, 326-365. 
Table 2: $\rho=0.2$, linear

\begin{tabular}{ll|ccc|rccc}
\hline \hline \multicolumn{2}{l}{ Productivity $=\omega_{i t}$} & Mean & Std & RMSE & $\omega_{i t}+a_{i}$ & Mean & Std & RMSE \\
\hline \multirow{2}{*}{ OLS } & $\beta_{l}$ & 1.2078 & 0.0234 & 0.5083 & 1.3400 & 0.0237 & 0.6404 \\
& $\beta_{k}$ & 0.3159 & 0.0216 & 0.0268 & 0.3797 & 0.0174 & 0.0816 \\
\hline \multirow{2}{*}{$\mathrm{FE}$} & $\beta_{l}$ & 1.1222 & 0.0309 & 0.4233 & & 1.1338 & 0.0319 & 0.4350 \\
& $\beta_{k}$ & 0.0309 & 0.0382 & 0.2718 & 0.1254 & 0.0295 & 0.1771 \\
\hline \multirow{2}{*}{$\mathrm{DP}$} & $\beta_{l}$ & 1.0602 & 0.6987 & 0.7860 & 1.0827 & 0.6944 & 0.7928 \\
& $\beta_{k}$ & 0.4111 & 0.4411 & 0.4549 & 0.4278 & 0.4433 & 0.4614 \\
\hline \multirow{2}{*}{ DPS } & $\beta_{l}$ & 1.2562 & 0.6749 & 0.8745 & & 1.2311 & 0.3501 & 0.6361 \\
& $\beta_{k}$ & 0.3026 & 0.0259 & 0.0260 & 0.3015 & 0.0275 & 0.0276 \\
\hline \multirow{2}{*}{ LP } & $\beta_{l}$ & 0.7001 & 0.0274 & 0.0274 & 0.7006 & 0.0286 & 0.0286 \\
& $\beta_{k}$ & 0.2866 & 0.1255 & 0.1262 & 0.4513 & 0.0483 & 0.1588 \\
\hline \multirow{2}{*}{ PIV } & $\beta_{l}$ & 0.7001 & 0.0274 & 0.0274 & 0.7006 & 0.0286 & 0.0286 \\
& $\beta_{k}$ & 0.3000 & 0.0337 & 0.0337 & 0.3015 & 0.0374 & 0.0374 \\
\hline
\end{tabular}

Note: I estimate the following models 1000 times and compute the mean, standard deviation and root mean squared error of the estimates. For each run, $N=250$ and $T=5$. FE refers to estimation via first differencing to remove $a_{i}$. DP and DPS are estimates based on the dynamic panel approach. DP only uses the differenced equation with levels as instruments while DPS uses the levels equation with differenced variables as instruments. LP corresponds to Levinsohn and Petrin (2003) where $\beta_{l}$ is estimated in the first stage. PIV is the approach proposed in the paper and shares the first stage of LP (hence the estimates for $\beta_{l}$ under LP and PIV are identical). The true values for the coefficients of interest are $\beta_{l}=0.7$ and $\beta_{k}=0.3$ 
Table 3: $\rho=0.2$, nonlinear

\begin{tabular}{ll|ccc|rccc}
\hline \hline \multicolumn{2}{l}{ Productivity $=\omega_{i t}$} & Mean & Std & RMSE & $\omega_{i t}+a_{i}$ & Mean & Std & RMSE \\
\hline OLS & $\beta_{l}$ & 1.2045 & 0.0233 & 0.5050 & 1.3395 & 0.0237 & 0.6400 \\
& $\beta_{k}$ & 0.3105 & 0.0217 & 0.0241 & 0.3784 & 0.0174 & 0.0803 \\
\hline FE & $\beta_{l}$ & 1.1336 & 0.0308 & 0.4347 & 1.1462 & 0.0319 & 0.4474 \\
& $\beta_{k}$ & 0.0181 & 0.0388 & 0.2845 & 0.1179 & 0.0300 & 0.1846 \\
\hline DP & $\beta_{l}$ & 1.0743 & 0.6970 & 0.7911 & 1.1145 & 0.6891 & 0.8041 \\
& $\beta_{k}$ & 0.4172 & 0.4591 & 0.4738 & 0.4248 & 0.4427 & 0.4600 \\
\hline \multirow{2}{*}{ DPS } & $\beta_{l}$ & 1.3049 & 0.6693 & 0.9021 & 1.3290 & 0.3533 & 0.7214 \\
& $\beta_{k}$ & 0.3015 & 0.0256 & 0.0257 & 0.3017 & 0.0260 & 0.0261 \\
\hline \multirow{2}{*}{ LP } & $\beta_{l}$ & 0.7001 & 0.0274 & 0.0274 & 0.7006 & 0.0286 & 0.0286 \\
& $\beta_{k}$ & 0.2847 & 0.1420 & 0.1428 & 0.4596 & 0.0353 & 0.1635 \\
\hline \multirow{2}{*}{ PIV } & $\beta_{l}$ & 0.7001 & 0.0274 & 0.0274 & 0.7006 & 0.0286 & 0.0286 \\
& $\beta_{k}$ & 0.3000 & 0.0330 & 0.0330 & 0.3020 & 0.0371 & 0.0371 \\
\hline
\end{tabular}

See note in Table 2. 
Table 4: $\rho=0.8$, linear

\begin{tabular}{ll|ccc|rccc}
\hline \hline \multicolumn{2}{l}{ Productivity $=\omega_{i t}$} & Mean & Std & RMSE & $\omega_{i t}+a_{i}$ & Mean & Std & RMSE \\
\hline OLS & $\beta_{l}$ & 1.3216 & 0.0228 & 0.6220 & 1.3903 & 0.0231 & 0.6907 \\
& $\beta_{k}$ & 0.3904 & 0.0194 & 0.0925 & 0.3978 & 0.0162 & 0.0991 \\
\hline FE & $\beta_{l}$ & 1.0427 & 0.0318 & 0.3442 & 1.0450 & 0.0327 & 0.3465 \\
& $\beta_{k}$ & 0.2045 & 0.0309 & 0.1003 & 0.2307 & 0.0251 & 0.0737 \\
\hline DP & $\beta_{l}$ & 1.0768 & 0.6870 & 0.7835 & 1.0819 & 0.6781 & 0.7783 \\
& $\beta_{k}$ & 0.4020 & 0.3983 & 0.4111 & 0.4162 & 0.4290 & 0.4444 \\
\hline \multirow{2}{*}{ DPS } & $\beta_{l}$ & 0.6978 & 0.1942 & 0.1942 & 0.7522 & 0.1829 & 0.1902 \\
& $\beta_{k}$ & 0.2987 & 0.0482 & 0.0483 & 0.2982 & 0.0480 & 0.0480 \\
\hline \multirow{2}{*}{ LP } & $\beta_{l}$ & 0.7000 & 0.0275 & 0.0275 & 0.7005 & 0.0286 & 0.0286 \\
& $\beta_{k}$ & 0.2645 & 0.1363 & 0.1409 & 0.3196 & 0.1152 & 0.1168 \\
\hline \multirow{2}{*}{ PIV } & $\beta_{l}$ & 0.7000 & 0.0275 & 0.0275 & 0.7005 & 0.0286 & 0.0286 \\
& $\beta_{k}$ & 0.2966 & 0.0639 & 0.0640 & 0.2977 & 0.0636 & 0.0637 \\
\hline
\end{tabular}

See note in Table 2. 
Table 5: $\rho=0.8$, nonlinear

\begin{tabular}{ll|ccc|rccc}
\hline \hline \multicolumn{2}{l}{ Productivity $=\omega_{i t}$} & Mean & Std & RMSE & $\omega_{i t}+a_{i}$ & Mean & Std & RMSE \\
\hline OLS & $\beta_{l}$ & 1.2630 & 0.0410 & 0.5645 & 1.3634 & 0.0346 & 0.6643 \\
& $\beta_{k}$ & 0.3489 & 0.0211 & 0.0532 & 0.3842 & 0.0182 & 0.0862 \\
\hline FE & $\beta_{l}$ & 1.0988 & 0.0577 & 0.4030 & 1.1055 & 0.0577 & 0.4096 \\
& $\beta_{k}$ & 0.1031 & 0.0367 & 0.2003 & 0.1658 & 0.0283 & 0.1371 \\
\hline DP & $\beta_{l}$ & 1.0334 & 0.6858 & 0.7626 & 1.0550 & 0.6861 & 0.7725 \\
& $\beta_{k}$ & 0.3959 & 0.3767 & 0.3887 & 0.3946 & 0.3707 & 0.3826 \\
\hline \multirow{2}{*}{ DPS } & $\beta_{l}$ & 0.9601 & 0.5256 & 0.5864 & 0.9704 & 0.2848 & 0.3927 \\
& $\beta_{k}$ & 0.3040 & 0.0303 & 0.0306 & 0.2998 & 0.0349 & 0.0349 \\
\hline \multirow{2}{*}{ LP } & $\beta_{l}$ & 0.7003 & 0.0281 & 0.0281 & 0.7005 & 0.0286 & 0.0286 \\
& $\beta_{k}$ & 0.2937 & 0.0480 & 0.0484 & 0.3929 & 0.0607 & 0.1110 \\
\hline \multirow{2}{*}{ PIV } & $\beta_{l}$ & 0.7003 & 0.0281 & 0.0281 & 0.7005 & 0.0286 & 0.0286 \\
& $\beta_{k}$ & 0.3010 & 0.0486 & 0.0486 & 0.3015 & 0.0490 & 0.0490 \\
\hline
\end{tabular}

See note in Table 2. 